\title{
Polyamines: fundamental characters in chemistry and biology
}

\author{
E. Agostinelli • M. P. M. Marques • R. Calheiros • \\ F. P. S. C. Gil - G. Tempera $\cdot$ N. Viceconte $\cdot$ \\ V. Battaglia $\cdot$ S. Grancara $\cdot$ A. Toninello
}

Received: 1 August 2009/Accepted: 8 October 2009/Published online: 15 December 2009

(C) Springer-Verlag 2009

\begin{abstract}
Polyamines are small cationic molecules required for cellular proliferation and are detected at higher concentrations in most tumour tissues, compared to normal tissues. Agmatine (AGM), a biogenic amine, is able to arrest proliferation in cell lines by depleting intracellular polyamine levels. It enters mammalian cells via the polyamine transport system. Agmatine is able to induce oxidative stress in mitochondria at low concentrations (10 or $100 \mu \mathrm{M}$ ), while at higher concentrations (e.g. 1-2 mM) it does not affect mitochondrial respiration and is ineffective in inducing any oxidative stress. As this effect is strictly correlated with the mitochondrial permeability transition induction and the triggering of the pro-apoptotic pathway, AGM may be considered as a regulator of this type of cell death. Furthermore, polyamine transport is positively
\end{abstract}

E. Agostinelli · G. Tempera - N. Viceconte

Department of Biochemical Sciences, Biology and Molecular Pathology Institutes, SAPIENZA University of Rome and CNR, Piazzale Aldo Moro 5, 00185 Rome, Italy

E. Agostinelli $(\bowtie)$

Department of Biochemical Sciences,

SAPIENZA University of Rome, Piazzale Aldo Moro 5,

00185 Rome, Italy

e-mail: enzo.agostinelli@uniroma1.it

M. P. M. Marques - R. Calheiros

Molecular Physical-Chemistry,

R\&D Unit University of Coimbra, Coimbra, Portugal

F. P. S. C. Gil

CEMDRX, Physics Department,

University of Coimbra, Coimbra, Portugal

V. Battaglia $\cdot$ S. Grancara $\cdot$ A. Toninello

Department of Biological Chemistry, University of Padua,

35121 Padua, Italy correlated with the rate of cellular proliferation. By increasing the expression of antizyme, a protein that inhibits polyamine biosynthesis and transport, AGM also exhibits a regulatory effect on cell proliferation. Methylglyoxal bis(guanylhydrazone) (MGBG), a competitive inhibitor of S-adenosyl-L-methionine decarboxylase, displaying anticancer activity, is a structural analogue of the natural polyamine spermidine. MGBG has been extensively studied, preclinically as well as clinically, and its anticancer activity has been attributed to the inhibition of polyamine biosynthesis and also to its effect on mitochondrial function. Numerous findings have suggested that MGBG might be used as a chemotherapeutic agent against cancer.

Keywords Biogenic polyamines .

Quantum mechanical calculations · Agmatine - MGBG ·

Structure-activity relationships $\cdot$ Mitochondria

$\begin{array}{ll}\text { Abbreviations } \\ \text { ADC } & \text { Arginine decarboxylase } \\ \text { AGM } & \text { Agmatine (4-(aminobutyl)guanidine) } \\ \text { AO-Agm } & N \text {-(3-aminoxy-propyl)-guanidin } \\ \text { BSAO } & \text { Bovine serum amine oxidase } \\ \text { DFMO } & \text { (D, L)-2-(difluoromethyl)ornithine } \\ \text { EMT } & \text { Extraneuronal monoamine transporter } \\ \text { GAPA } & N \text {-(3-aminopropoxy)guanidine } \\ \text { INS } & \text { Inelastic neutron scattering } \\ \text { IR } & \text { Infrared spectroscopy } \\ \text { MDR } & \text { Multidrug resistant } \\ \text { MGBG } & \text { Methylglyoxal bis(guanylhydrazone) } \\ \text { MPT } & \text { Mitochondrial permeability transition } \\ \text { NGPG } & N \text {-(3-guanidino-propoxy)guanidine } \\ \text { OCT2 } & \text { Organic cation transporter 2 } \\ \text { ODC } & \text { Ornithine decarboxylase }\end{array}$




\section{PAs Polyamines}

RLM Rat liver mitochondria

ROS Reactive oxygen species

SAMDC S-adenosyl-L-methionine decarboxylase

SAR's Structure-activity relationships

SSAT Spermidine/spermine $N$-acetyltransferase

\section{Introduction}

The natural polyamines (putrescine, spermidine, spermine and related structures) are formed from the decarboxylation products of ornithine and S-adenosyl-methionine in nearly all eukaryotic cells. The polyamine biosynthetic pathway is very active during the growth of various cancer cells. In fact, polyamines are often present at high concentrations in rapidly dividing tumour cells and growing tissues. Motives for these increased levels include enhanced putrescine synthesis from ornithine by ornithine decarboxylase (ODC) and increased uptake of polyamines (Marton and Pegg 1995). ODC, one of the most highly regulated eukaryotic enzymes, is a proto-oncogene that is significantly elevated in animal tumours (Scalabrino and Ferioli 1981, 1982) and is the first and rate-limiting enzyme of polyamines biosynthesis. Polyamines, being essential for eukaryotic cell growth and differentiation, are known to be implicated in a bewildering number of cellular roles as well as in maintaining the native structure of several biological macromolecules while affecting the activity of others through tightly regulated concentration-dependent processes (Gerner and Meyskens 2004; Bachrach 2005; Seiler and Raul 2005; Gupta et al. 2005; Nasizadeh et al. 2005; Manni et al. 2006; Pegg and Feith 2007).

Moreover, several findings suggest that the deregulation of polyamine metabolism may induce apoptosis (Seiler and Raul 2005). Apart from being of vital importance for the propagation and viability of most cells, the natural polyamines spermidine and spermine are also the source of cytotoxic metabolites (Agostinelli et al. 2004, 2006). In fact, the oxidative deamination of spermine by bovine serum amine oxidase (BSAO: EC 1.4.3.6) generates hydrogen peroxide and aldehyde(s), which are able to induce both apoptotic and non-apoptotic cell death (Fig. 1) (Agostinelli et al. 2007, 2009).

Therefore, the primary role of polyamines in regulating proliferation and cellular death have led scientists to investigate the role of these molecules at the level of mitochondria and multifunctional organelles participating in a range of cellular processes such as energy production, proliferation, senescence and death (Goldenthal and Marin-Garcia 2004). Mitochondria apparently lack a polyamine biosynthetic pathway, although substantial quantities of spermine and

\section{REACTION SCHEME}

\author{
$\mathrm{NH}_{2}\left(\mathrm{CH}_{2}\right)_{3} \mathrm{NH}\left(\mathrm{CH}_{2}\right)_{4} \mathrm{NH}\left(\mathrm{CH}_{2}\right)_{3} \mathrm{NH}_{2}+2 \mathrm{O}_{2}+2 \mathrm{H}_{2} \mathrm{O}$ \\ (spermine)

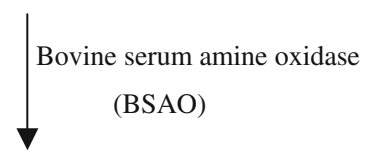 \\ $\mathrm{CHO}\left(\mathrm{CH}_{2}\right)_{2} \mathrm{NH}\left(\mathrm{CH}_{2}\right)_{4} \mathrm{NH}\left(\mathrm{CH}_{2}\right)_{2} \mathrm{CHO}+2 \mathrm{NH}_{3}+2 \mathrm{H}_{2} \mathrm{O}_{2}$ \\ ( dialdehyde )
}

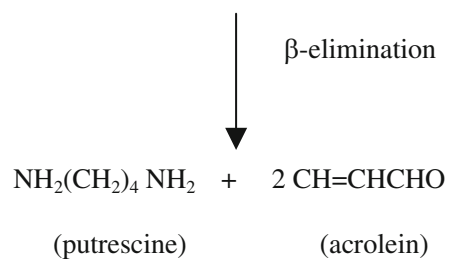

Fig. 1 Reaction scheme for spermine oxidation in the presence of BSAO

spermidine have been detected in the mitochondrial matrix and a specific mitochondrial polyamine transporter has been detected (Toninello et al. 2004). Moreover, a specific transport system has also been described for the diamine agmatine, a molecule belonging to the polyamine family (Salvi et al. 2006). Arginine decarboxylase (ADC) converts arginine to agmatine (AGM), which is a dication at physiological $\mathrm{pH}$. Agmatine inhibits proliferation by suppressing intracellular polyamine levels. The main target of polyamines seems to be the mitochondrial permeability transition (MPT) pore, a structure involved in mitochondria-mediated cell death, where they exert a protective role by both direct and indirect mechanisms (Toninello et al. 2004). Polyamines are also known to regulate the activity of pyruvate dehydrogenase in liver mitochondria, suggesting their primary role in regulating the energy metabolism (Pezzato et al. 2009). Polyamines are also involved in numerous relevant pathologies, such as cancer and immunological, neurological and gastrointestinal diseases.

Guanidino-containing drugs as methylglyoxal bis (guanylhydrazone) (MGBG) were demonstrated to have antitumour properties and have therefore been exposed to intensive preclinical and clinical evaluations (Jänne et al. 1991). Several findings suggest that MGBG uses the polyamine transport system to enter cells, and depletion of spermine and spermidine by treatment with (D, L)-2(difluoromethyl)ornithine (DFMO) increases the uptake of MGBG. In fact, in association with DFMO, MGBG showed synergistic responses in childhood leukaemia and in P388 leukaemia in mice (Nakaike et al. 1988). This cytotoxicity is probably due to MGBG's ability to prevent mitochondrial spermine flux, since spermine uptake is determined by the sum of two processes: membrane 
binding and electrophoretic matrix transport (Dalla Via et al. 1996). These observations strongly suggest a renewed use of MGBG, with an appropriate schedule, as an antineoplastic drug.

\section{Polyamines as essential biomolecules}

Polyamines such as-putrescine $\left(\mathrm{H}_{2} \mathrm{~N}\left(\mathrm{CH}_{2}\right)_{4} \mathrm{NH}_{2}\right)$, spermidine $\left(\mathrm{H}_{2} \mathrm{~N}\left(\mathrm{CH}_{2}\right)_{3} \mathrm{NH}\left(\mathrm{CH}_{2}\right)_{4} \mathrm{NH}_{2}\right)$ and spermine $\left(\mathrm{H}_{2} \mathrm{~N}\right.$ $\left(\mathrm{CH}_{2}\right)_{3} \mathrm{NH}\left(\mathrm{CH}_{2}\right)_{4} \mathrm{NH}\left(\mathrm{CH}_{2}\right)_{3} \mathrm{NH}_{2}$ ) (Fig. 2)-are ubiquitous in cells of higher organisms, and result from the decarboxylation of ornithine. Under physiological conditions, these linear amines (PAs) are totally protonated and behave as natural polycations, capable of interacting with both DNA and RNA, therefore inducing conformational transitions and affecting nucleic acid function (Ouameur and Tajmir-Riahi 2004; Ruiz-Chica et al. 2003; N'soukpoKossi et al. 2008; Wallace 2003). Actually, it has been shown that biogenic polyamines cause DNA condensation in both isolated DNA (Patel and Anchoroquy 2006) and chromatin (Todd et al. 2008). However, the exact nature of the polyamine-DNA interaction is not clearly established and is the subject of great controversy. Putrescine, spermidine and spermine are known to bind through the minor and major grooves of double strand DNA, while electrostatic attractions between these polycations and the DNA phosphate backbone also occur (Ouameur and TajmirRiahi 2004).

On account of absolute polyamine requirement for cell growth, interference with polyamine biosynthesis can be a rather promising therapeutic approach against neoplastic diseases (Wallace 2007; Casero and Marton 2007).
Polyamine concentrations are high in growing tissues such as tumours, for example, breast and colon cancer (Heby and Persson 1990), if compared to normal tissues. In fact, polyamines have been proposed as biochemical markers of neoplasia, since high polyamine concentrations are found in physiological fluids of patients with malignant diseases.

Polyamine analogues (e.g. oligoamines such as $N$-alkylated biogenic polyamines, Fig. 3), in particular, have been shown to deplete cells of their natural polyamines, by various mechanisms affecting cellular polyamine homeostasis (Carew et al. 2008; Huang et al. 2006). These analogues appear to affect the regulatory pathways associated to polyamine biosynthesis, catabolism and transport (Pegg 2008; Casero and Marton 2007), thus interfering with polyamines intracellular concentration. In fact, although polyamine analogues are recognised by the cell as natural polyamines, they are unable to substitute for these in their growth promoting role (Ha et al. 1998). Some of these compounds have displayed exceptional efficacy, coupled to surprisingly low toxicity, in animal tumour models (e.g. in breast and lung cancers), being already in clinical treatment trials. However, despite the high number of studies on this subject, the molecular basis of the polyamine analogues' anti-neoplastic activity is still scarcely identified, as there is a gap in the knowledge of their structural preferences in relation to uptake and activity.

Furthermore, amines are known to be suitable chelating ligands for transition metal ions such as $\mathrm{Pt}(\mathrm{II})$ or $\mathrm{Pd}(\mathrm{II})$, yielding stable and usually water soluble coordination compounds, often active as pharmacological agents (e.g. anticancer drugs) as described above. Linear aliphatic amines, specifically, are recognised to have a high conformational freedom (Batista de Carvalho et al. 1999;
Fig. 2 Schematic representation of the most stable calculated geometries for some biogenic polyamines: neutral and cationic forms of putrescine (a); totally protonated species of spermidine (b) and spermine (c) (a)

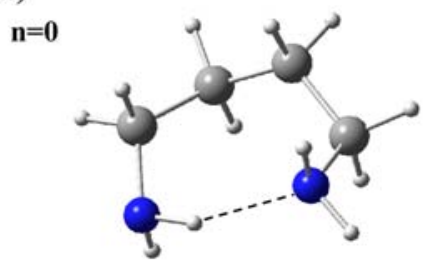

(b)

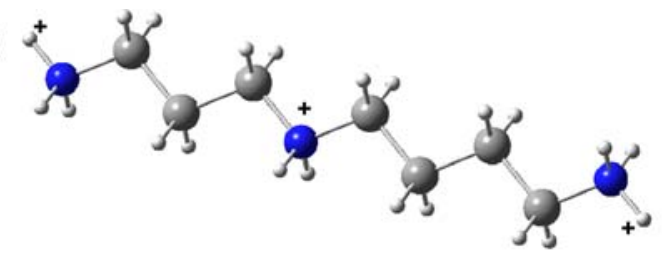

(c)

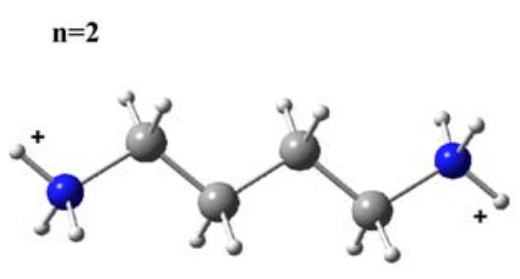

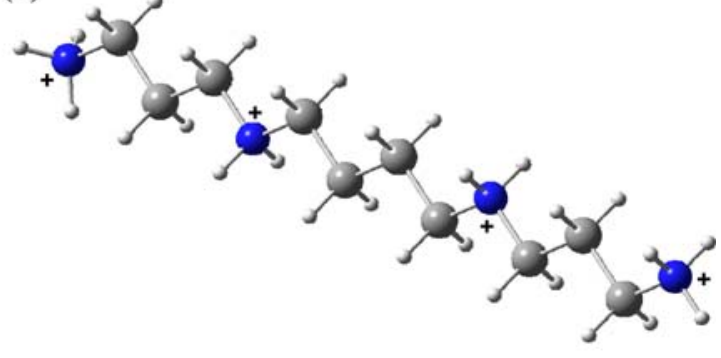

$\mathrm{C} \odot \mathrm{N}, \mathrm{H}$ 


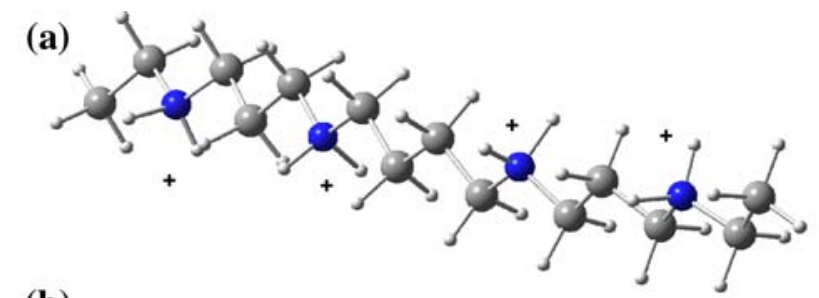

(b)

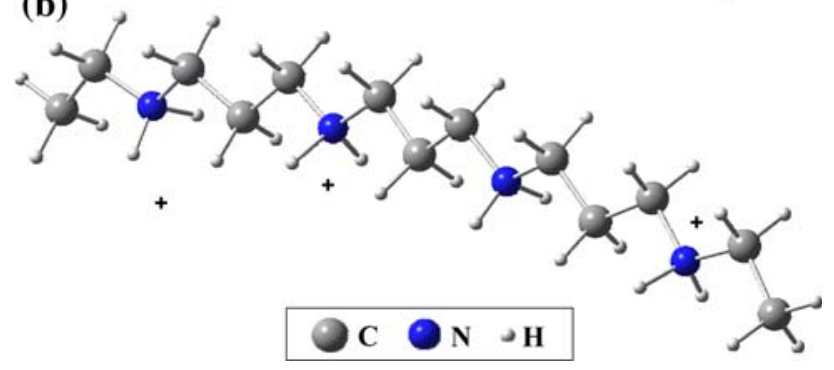

Fig. 3 Schematic representation of the most stable calculated geometries for some spermine analogues (totally protonated species): BES (N1,N12-diethyl spermine tetra-hydrochloride) (a) and DENSPM (N1,N11-diethylnorspermine tetrahydrochloride) (b)

Marques and Batista de Carvalho 2000; Marques et al. 2002a, b, 2006, 2007; Marques and Batista de Carvalho 2007; Amorim da Costa et al. 2002, 2003, 2004; Amado et al. 2004) and may be designed to display high flexibility and polidenticity, which constitutes an advantage for an effective interaction with biological receptors. In addition, the dual hydrophilic-lipophilic character of the polyamine ligands, comprising cationic amine groups (both primary and secondary) and variable length hydrophobic alkyl linkers, will possibly lead to an enhanced cellular uptake. This hydrophilic-lipophilic balance depends on the relationship between the length of the carbon bridging chains and the number of amine moieties. In addition, the presence of $\mathrm{NH}$ groups will favour the molecular recognition of the polyamines and polyamine-based agents by the polyphosphate backbone of DNA and related targets (e.g. purine bases), due to the formation of $(\mathrm{N}) \mathrm{H} \cdots \mathrm{O}$ hydrogen bonds (Liu et al. 2006).

Linkage of linear polyamines to previously tested anticancer agents was found to lead to a higher cytotoxic effect (Fogel-Petrovic et al. 1996, 1997; Ichimura et al. 1998). In some cases, they can even enhance the efficacy of the long used first-generation drug cisplatin. Over the last two decades, several studies on the cytotoxic properties of transition metal complexes (e.g. Pt(II) and Pd(II)) with aliphatic polyamines have been carried out, aiming at obtaining new anticancer, third-generation drugs, displaying tissue specificity and enhanced efficacy relative to the clinically used compounds. In particular, a group of cisplatin-like compounds containing polyamine bridging ligands (putrescine, spermidine or spermine) have proven to display novel antitumour properties (Qu et al. 2000;
Marques et al. 2002a, b; Teixeira et al. 2004; Harris et al. 2006; Komeda et al. 2006; Fiuza et al. 2006) due to the formation of a new type of DNA adducts, through long-distance intra- and interstrand cross-links or by noncovalent interactions, not available to the conventional alkylating agents (e.g. triplatinum BBR3464) (Qu et al. 2000; Pratesi et al. 1999) and Triplatinum NC, (Komeda et al. 2006; Harris et al. 2005).

\section{Conformational behaviour versus biological activity}

The activity of a biologically relevant compound does not rely solely on its chemical properties, but it is also dependent on its conformational preferences, that must be accurately determined for a thorough understanding of its function and mechanisms of action. The highly sensitive structure-activity relationships (SAR's) that underlie and control polyamine function have been the target of intense research in the last two decades.

Vibrational spectroscopy-infrared (IR), Raman and inelastic neutron scattering (INS) - coupled to theoretical calculations (quantum mechanical methods), is a specially useful method for achieving this goal, since it yields valuable structural evidence based on the analysis of the spectral features associated to each chemical group within the molecule. Raman spectroscopy, in particular, has proved to be a reliable technique for the characterisation and conformational analysis of biochemically active molecules, since it provides unique fingerprint spectra specific for each compound (Marques et al. 2007). Actually, due to its non-invasiveness, high sensitivity and good reproducibility, this technique, which does not need any special sample preparation, is becoming a valuable tool in the fields of Biological and Medicinal Chemistry. INS spectroscopy, in turn, is particularly well suited to the study of hydrogenous compounds such as polyamines, which yields data complementary to the one obtained by either Raman or IR spectroscopies.

A complete conformational analysis of a particular polyamine can, therefore, be attained through its study by spectroscopic and theoretical approaches, which will yield the precise conformation of the molecule under study at the required conditions (for defined values of temperature, $\mathrm{pH}$ and/or ionic strength, e.g. physiological medium). This interplay between experimental and theoretical data is essential for a better understanding of the biochemical function of polyamines and polyamine-based systems at a molecular level, leading to the establishment of reliable SAR's that will hopefully allow to interpret the biological pathways in which these molecules are involved.

During the last few years, the biogenic tri- and tetramines spermidine and spermine have been investigated as to 
their conformational behaviour, as well as the homologous series of $\alpha, \omega$-diamines $\mathrm{H}_{2} \mathrm{~N}\left(\mathrm{CH}_{2}\right)_{n} \mathrm{NH}_{2} \quad(n=2-10$, $n=12) \quad$ (which comprise putrescine $(n=4)$-Fig. 2) (Batista de Carvalho et al. 1999; Marques and Batista de Carvalho 2000; Marques et al. 2002a, b, 2006, 2007; Marques and Batista de Carvalho 2007; Amorim da Costa et al. 2002, 2003, 2004). The conformational preferences of these linear alkylamines were found to depend on several factors, from steric, dipolar and hyperconjugative effects to the balance between intra- and intermolecular interactions. This kind of systems are characterised by a high conformational freedom (putrescine, for instance, can adopt six different stable conformations for its physiological, dicationic state $\left.\left[\mathrm{H}_{3} \mathrm{~N}\left(\mathrm{CH}_{2}\right)_{4} \mathrm{NH}_{3}\right]^{2+}\right)$, and by an interdependence of the particular effects due to the electronegativity and electron lone-pairs of the nitrogen atoms. These lead to the formation of intra- and intermolecular hydrogen bonds (e.g. $\mathrm{R}-\mathrm{HN}-\mathrm{H} \cdots \mathrm{NH}_{2}-\mathrm{R}$ ), which determine the solid-state conformational behaviour of the amines and may give rise to either infinite chain polymeric forms or to dimeric species (e.g. for diaminoethane) (Amado et al. 2004).

Moreover, since biogenic amines are physiological polycations, the effect of protonation on their conformational preferences has been investigated, by studying the corresponding vibrational pattern as a function of $\mathrm{pH}$. Protonation of all the nitrogen atoms, yielding charged species $(\mathrm{q}=2+, 3+$ or $4+$ for putrescine, spermidine and spermine, respectively) hinders the formation of either $(\mathrm{N}) \mathrm{H} \cdots: \mathrm{N}$ or $(\mathrm{C}) \mathrm{H} \cdots: \mathrm{N}$ intramolecular close contacts, and can even lead to $(\mathrm{N}) \mathrm{H} \cdots \mathrm{H}(\mathrm{N})$ and/or $(\mathrm{N}) \mathrm{H} \cdots \mathrm{H}(\mathrm{C})$ repulsive interactions within the molecule, drastically affecting its conformation. Therefore, the completely protonated, polycationic polyamines present in physiological media display a linear, totally extended, geometry (Fig. 2).

In short, the conformational characteristics of biogenic polyamines rely on a tight balance between the formation of stabilising intramolecular hydrogen-like bonds $((\mathrm{N}) \mathrm{H} \cdots: \mathrm{N}$ and/or $(\mathrm{C}) \mathrm{H} \cdots: \mathrm{N})$ and minimisation of steric and electrostatic repulsive interactions. While the former, intramolecular close contacts, are predominant in the unprotonated species, the intermolecular effects are the only ones taking place in the protonated forms. The conformational preferences of this kind of systems comprising different protonation sites (nitrogen atoms) are then clearly and expectedly ruled by the environment and $\mathrm{pH}$ conditions of the medium (e.g. type of tissue and nearest biomolecules or receptors).

\section{Structural properties of agmatine and its transport in cells}

The structural preferences of AGM were determined, both in the solid and in aqueous solution (for AGM's distinct protonation states), by Raman spectroscopy combined to theoretical calculations (Toninello et al. 2006) and the structural information thus gathered was analysed in order to explain the biological results simultaneously obtained in rat liver mitochondria (RLM) (Salvi et al. 2006), and hepatocytes. The effect of several structural parameters on the overall stability of the molecule was investigated, with special emphasis on the orientation of the guanidinium group relative to the aliphatic chain. As the expected structural changes due to N-protonation are clearly detected in the Raman pattern gathered for the aqueous solutions as a function of $\mathrm{pH}$, the protonation state of AGM can thus be easily and unequivocally determined through this spectroscopic technique.

A thorough structural characterisation of the most stable AGM geometries in aqueous solution, under different $\mathrm{pH}$ conditions, was achieved: the diprotonated (dipositive) species, at physiological $\mathrm{pH}$; the monoprotonated (monopositive) form, at alkaline $\mathrm{pH}$ (ca. 7.3-9.0); and the totally unprotonated (neutral) molecule, in strong alkaline medium (Fig. 4). The most stable physiological AGM structures were identified as being two dipositive species with a lower negative charge in the protonated aliphatic amino terminal relative to the monopositive or neutral forms of the molecule. Most probably, these are the conformations to be found in the biological fluids during AGM absorption from the diet, as well as in the cytosol, in mammalian cells, after AGM is transported across the plasma membrane. Consequently, these are prone to be the species that interact with the mitochondrial membrane and have a marked effect on MPT. The predominant monocationic AGM species, which may also exist in particular microenvironments, and the most stable neutral conformations, responsible for interaction with hydrophobic sites in vivo, were also determined. As mentioned above, although a transport system for AGM in hepatocytes has been individualised, no specific mechanism, present in the plasma membrane, has been, to this date, characterised at the molecular level. However, there are several models reported in the literature aimed to give information about this process. In particular, it has been suggested tha AGM may be transported in human cell lines derived from embryonic kidney by the monoamine systems extraneuronal monoamine transporter (EMT) and organic cation transporter 2 (OCT2) (Cabella et al. 2001). Thus, monoprotonated AGM would be the sole substrate for the cited transporters. However, in considering the kinetic characteristics of the transport mechanism, which is most likely an electroneutral one, AGM should not be transported in the monoprotonated form, which has a high dipole moment. Instead, it is proposed that the amine is taken up in the totally unprotonated, uncharged form. If the transport across the plasma membrane takes place with the neutral molecule, the transport across the mitochondrial 
Fig. 4 Schematic representation of the most stable calculated structures for agmatine, in aqueous solution, in its distinct protonation states (unprotonated (neutral), monoand diprotonated, $n=0,1$ and 2 , respectively). (The most abundant physiological form of agmatine is the dicationic one.)
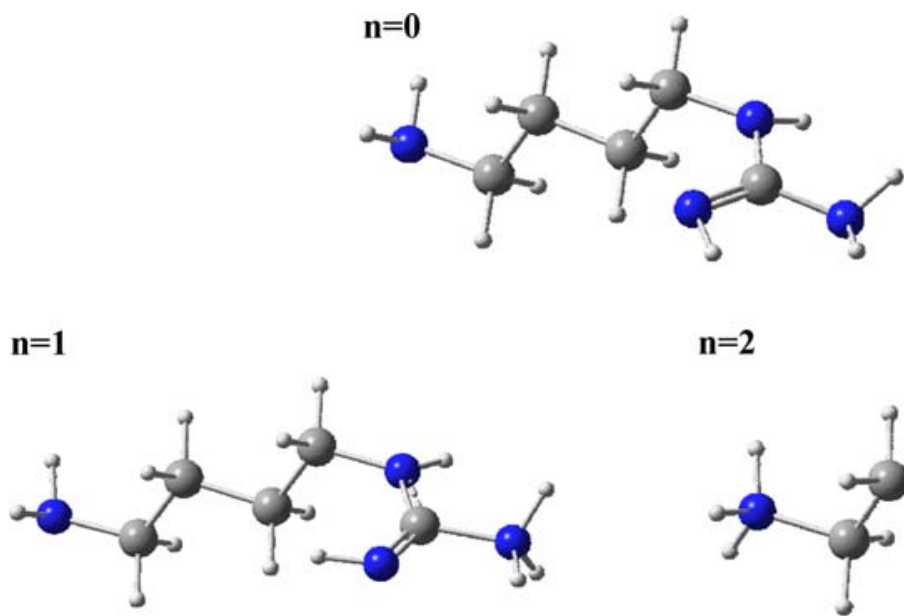

$\mathbf{n}=\mathbf{2}$

\section{$\rightarrow \mathrm{C} \odot \mathrm{N} \cdot \mathrm{H}$}

membrane exhibits a different behaviour. In this regard, as above mentioned, there was an alternative between a channel, in which the amine should be transported as a diprotonated molecule, or by a single centre-gated pore for the monoprotonated form (Salvi et al. 2006). The experiments using inhibition polyamine analogues strongly suggested that the latter hypothesis is the most valid one (Grillo et al. 2007). The structural results obtained for AGM showed that the diprotonated species has a dipole moment of about one-half that of the monopositive amine (Toninello et al. 2006). Thus, if the transport of AGM in mitochondria requires a high dipole moment, this implies that this amine is carried as a monovalent molecule, in agreement with the results gathered for the AGM analogues (Grillo et al. 2007).

This kind of structure-activity studies is essential for the understanding of AGM's diverse biological roles, such as its effect on MPT and its specific transport mechanisms, as well as for the development of new AGM-based therapeutic strategies (e.g. against drug addiction, pain-killing or tumour suppressing).

\section{The role of agmatine as a regulator of mitochondrial function}

Agmatine (4-(aminobutyl)guanidine), AGM, (Figs. 4, 5a) is a polyamine produced by decarboxylation of L-arginine, which displays a wide range of physiological functions (Grillo and Colombatto 2004), form neurotransmitter or neuromodulator ( $\mathrm{Li}$ et al. 1994; Raasch et al. 2001) to stimulator of insulin release and tumour suppressor agent (Satriano et al. 1998,1999; Dudkowska et al. 2003; Higashi et al. 2004; Gardini et al. 2001). Similar to the other biogenic polyamines, the activity of AGM as a biological effector and its specific transport mechanisms across plasma and mitochondrial membranes are strongly dependent on its structural preferences (Fig. 6). AGM is taken up in hepatocytes by both a high-affinity transport system and a low-affinity mechanism. The high-affinity system also transports putrescine, but not spermidine, spermine or cationic amino acids. The rate of AGM uptake is increased in cells deprived of polyamines by the effect of the ODC inhibitor, DFMO (Cabella et al. 2001). In fact, DFMO is presently undergoing clinical evaluations as a chemoprevention agent (Gerner et al. 2007).

It is to consider that the transport of AGM in hepatocytes is followed by its transport in isolated liver mitochondria. In fact, AGM is transported in these mitochondria by an energy-dependent mechanism driven by the electrical membrane potential $(\Delta \Psi)$. Although this process exhibits a strict electrophoretic behaviour, qualitatively similar to those of polyamines, AGM is most probably taken up by a specific transporter as divalent polyamines and cationic amino acids are completely ineffective in inhibiting AGM transport. Flux-voltage analysis and the determination of activation enthalpy are consistent with the hypothesis that the mitochondrial AGM transporter is a channel or a single-binding centre-gated pore. As discussed below, the theoretical studies on AGM structure in biological environments have permitted to clarify this point. As AGM is transported in liver mitochondria in a dose-dependent manner (Salvi et al. 2006), the effects of different amounts of amine have been evaluated at the level of the MPT induction. In this regard, it should be emphasised that low concentrations of AGM $(10-100 \mu \mathrm{M})$ are able to amplify the $\mathrm{Ca}^{2+}$-induced MPT, by means of an oxidative stress pathway. This process is triggered by the reaction products of AGM oxidation catalysed by a mitochondrial amino oxidase recently 
(a)
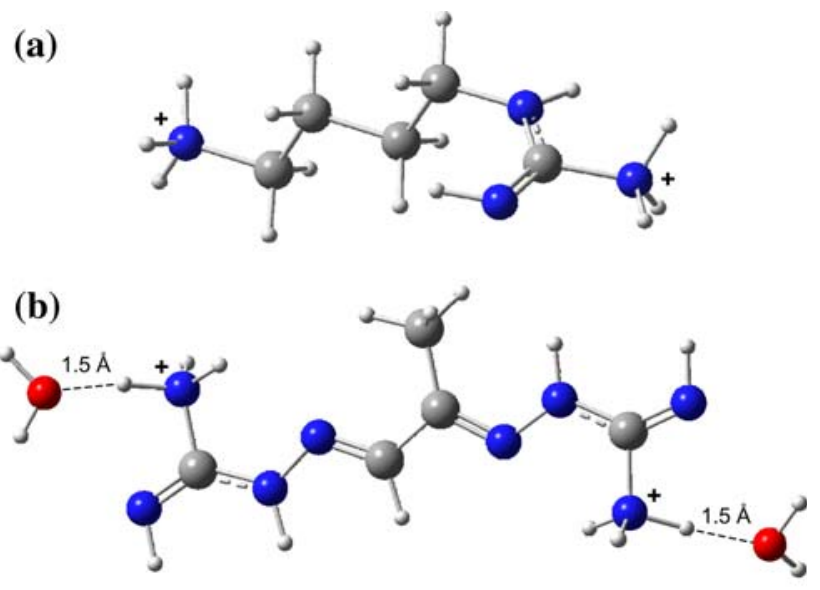

$\mathrm{C} \bigcirc \mathrm{O} \bigcirc \mathrm{N} \sim \mathrm{H}$

Fig. 5 Schematic representation of the most stable calculated structures, at physiological conditions, for agmatine (a) and MGBG (b). The $(\mathrm{N}) \mathrm{H} \cdots \mathrm{O}(\mathrm{H})$ interactions with the solvent molecules (water) are shown for diprotonated MGBG

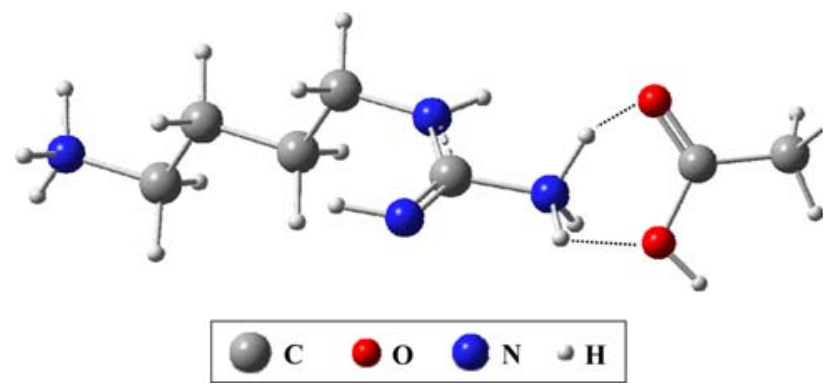

Fig. 6 Schematic representation of the possible interaction between acidic aminoacid terminals (e.g. from a transport protein) and the most stable structure of agmatine at physiological conditions (dicationic species)

discovered (Cardillo et al. 2009). Alternatively, by interacting with an iron sulphur centre of the respiratory chain, AGM can produce an imino radical and subsequently the superoxide anion, hydrogen peroxide and the hydroxyl radical (Battaglia et al. 2007). The importance of these AGM effects are related to the bioenergetic collapse and the triggering of the pro-apoptotic pathway, which follow them. However, it is also to stress that if AGM is present at higher concentrations (e.g. 1-2 mM), the picture of its action strongly changes. In fact, at these concentrations, AGM does not affect mitochondrial respiration and is ineffective in inducing oxidative stress and, consequently, the MPT. Moreover, it maintains the normal level of ATP synthesis and prevents the MPT induction by $\mathrm{Ca}^{2+}$ in the presence of phosphate. The inefficacy exhibited by AGM in inducing oxidative effects may be due to the antioxidant property that it exhibits at high concentrations, as was also demonstrated for polyamines. In these conditions, the amine can generate reactive oxygen species (ROS), as proposed above, but the amount of its still unreacted molecules may act as a scavenger, thus exhibiting selfprotection against the effects of ROS produced by both the amine and $\mathrm{Ca}^{2+}$ (Battaglia et al. 2007). This mechanism is similar to that previously proposed for spermine (Sava et al. 2006; Ha et al. 1998) and it is applicable when the targeted ROS is the hydroxyl radical. According to this model, unprotonated AGM reacts with $\mathrm{OH}^{*}$ to form dihydroxyaminobutyl-guanidine and, subsequently, guanidobutyric aldehyde is formed by spontaneous dehydration and hydrolysis (Sava et al. 2006). As previously suggested (Salvi et al. 2006), AGM transport in liver mitochondria could be mediated by a channel or a single-binding centregated pore, both displaying electrophoretic behaviour. The transport is non-competitively inhibited by the propargylamines while, in general, natural polyamines are ineffective as well as basic amino acids (Salvi et al. 2006). In order to gain further information on the origin of the AGM transporter, as well as on the reactive structure of AGM involved in the above mentioned processes, charge deficient AGM analogues, $N$-(3-aminoxy-propyl)-guanidine (AO-Agm), $N$-(3-aminopropoxy)guanidine (GAPA) and $\mathrm{N}$-(3-guanidino-propoxy)guanidine (NGPG) (Simonian et al. 2005) were investigated, evidencing an inhibition efficacy following the sequence NGPG $>$ AO-Agm $>$ GAPA. Since the positive charge of the analogues in this sequence, is approximately $1.5,1$ and 1.5 , respectively, their efficacy is not directly related to their charge (Grillo et al. 2007). Considering the mechanism proposed for the AGM transport (Salvi et al. 2006), these observations reflect the significant importance exhibited by the non-modified guanidine group for the transport inhibition, if compared with the charge-dependence, thus demonstrating that the transporter is a single-binding centre-gated pore rather than a channel.

\section{Biological function of anticancer methylglyoxal bis(guanylhydrazone)}

Methylglyoxal bis(guanylhydrazone) (Fig. 5b) is a polycation (at physiological conditions) containing two guanidinium groups, which acts as a competitive inhibitor of S-adenosyl-L-methionine decarboxylase (SAMDC), therefore, being involved in the biosynthesis of the biogenic polyamines spermidine and spermine. During the 1960 s, it attracted a considerable interest for its antineoplastic and strong cytotoxic effects, but it was discarded from therapeutical use due to its severe toxicity. However, subsequent clinical investigations have shown that particular administration conditions strongly reduce MGBG's toxicity while preserving its antitumoural action (Von Hoff 1994). In 
order to establish the relationship between MGBG's structural preferences and its biological action-in particular, its transport mechanism(s), binding to macromolecular structures and MPT prevention-a conformational analysis in aqueous solution, as a function of $\mathrm{pH}$, was carried out through theoretical methods (Marques et al. 2008). A complete geometry optimisation was carried out, considering the molecule's different protonation states, with particular emphasis on the diprotonated form, which is the major species under physiological conditions ( $\mathrm{pK}$ $(\mathrm{MGBG})=7.5$ and 9.2).

The most stable fully protonated MGBG geometry, largely predominant at $25^{\circ} \mathrm{C}(95.7 \%)$, displays a coplanar structure and a trans orientation of the two $(\mathrm{C}=) \mathrm{NH}$ groups relative to the $\mathrm{NH}_{3}{ }^{+}$moieties (Fig. 5b). Similarly, to the reported results on AGM (Toninello et al. 2006), which contains one $\mathrm{NH}_{3}{ }^{+}$terminal and a guanidinium moiety (Fig. 5), a trans location of the two imino NH's relative to the $\mathrm{NH}_{3}{ }^{+}$groups of the MGBG molecule is preferred over a cis orientation. Along with this predominant diprotonated species $(\mu=1.3 \mathrm{D})$, the MGBG monocationic form is also expected to exist at physiological conditions (Fig. 7), its relative populations (ca. 60\%: 40\%) being determined by the amine $\mathrm{pK}$ values of the molecule. Moreover, the very high dipole moment of this monoprotonated conformer ( $\mu=17.2 \mathrm{D})$ can favour its in vivo occurrence (polar medium). Consequently, these are proposed to be the main MGBG species present in biological fluids and inner cell compartments, responsible for the interaction with different macromolecular structures in vivo, leading to the known MGBG antitumoural effect.

\section{Conclusions}

When polyamines accumulate excessively within the cells, either due to very high extracellular amount or to deregulation of the systems controlling polyamine homeostasis,

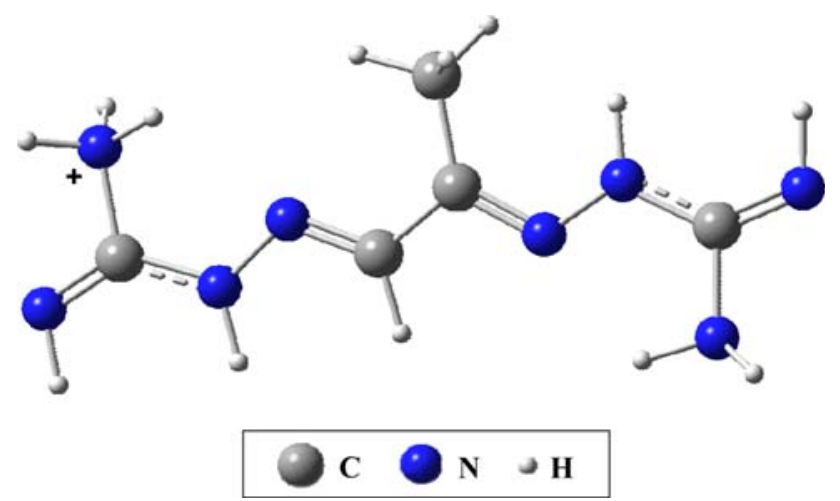

Fig. 7 Schematic representation of the most stable monoprotonated species of MGBG they can induce toxic effects. High intracellular levels of spermine, for instance, can cause an increase of its oxidative products inducing DNA damage and cell death for either necrosis or apoptosis. Therefore, the increase of in situ and in vivo formation of cytotoxic spermine metabolites can be important in inducing anti-tumoural effects (Agostinelli et al. 2004; Averill-Bates et al. 2005). Our findings show that purified BSAO and spermine may induce cell death in wild type and multidrug resistant (MDR) human M14 melanoma, B16 melanoma and adenocarcinoma LoVo cells, by both apoptosis and necrosis, as well as cytotoxicity (loss of cell proliferation) (Agostinelli et al. 2006, 2009; Averill-Bates et al. 2008). More recently, polyamines have also been implicated in events inherent to genetically programmed cell death (Seiler and Raul 2005; Schipper et al. 2000).

Arginine decarboxylase is responsible for the conversion of arginine to AGM. Intracellular concentrations of AGM are different among organs, with high levels of synthesis and expression in kidney, liver, brain and adrenals, which keep up the levels of ADC activity (Lortie et al. 1996). However, AGM has been detected in several organs that do not exhibit ADC activity at concentrations several orders of magnitude higher than those found in the kidney, liver or brain (Lortie et al. 1996), suggesting that circulating AGM could have both paracrine and endocrine effects. AGM can bring about both the production of NO as well as the intracellular concentrations of polyamines. Investigations on the effects of AGM on NO generation yielded contradictory results, while AGM is demonstrated to inhibit proliferation by suppressing intracellular polyamine levels (Babal et al. 2001; Dudkowska et al. 2003; Vargiu et al. 1999), by induction of antizyme (Satriano et al. 1998). It also induces spermidine/spermine $N$-acetyltransferase (SSAT) activity (Vargiu et al. 1999) in some cells types, which would promote the back-conversion of higher order (more highly charged) to lower order polyamines (Vargiu et al. 1999). These and other processes reduce intracellular polyamine pool and suppress growth. In agreement with these studies, it was found that AGM administration suppresses ODC activity in numerous cell lines and is also able to reduce intracellular polyamines by upregulation of SSAT (Vargiu et al. 1999).

Natural occurring polyamine metabolism is one of the main targets of $\mathrm{MGBG}$, which reduces the intracellular polyamine content through induction of SSAT, involved in polyamine catabolism, (Regenass et al. 1992) and inhibition of SAMDC, and therefore, important for polyamine synthesis (Williams-Ashman and Schenone 1972). Treatment of cells with MGBG results in inhibition of cell growth, an effect that can be reversed by exogenous spermidine. Regarding its length, MGBG is similar in structure to spermidine and thus being able to interfere with 
polyamine production. Although it is supposed to bind to DNA, since it is not completely protonated at physiological $\mathrm{pH}$ this binding is likely to be weaker as compared to the biogenic polyamines. However, several investigations evidence a clear MGBG anti-tumour activity, probably due to the low induced intracellular polyamine pool (Von Hoff 1994). Although the mechanisms underlying these antiproliferative and cytotoxic effects, they are still not well clarified. They might be linked to the formation of ROS upon MGBG oxidation, or to interaction with polyamine metabolism and mitochondrial function. In fact, various studies showed that MGBG induces evident damage to mitochondria with ultrastructural alterations, such as loss of cristae and matrix components as well as metabolic impairment, that are indicative of failure of mitochondrial integrity (Pleshkewych et al. 1980).

In conclusion, numerous studies on the MGBG transport across the plasma membrane of several cell lines report that this compound shares the same transporter with biogenic polyamines. Attending to the dicationic nature of AGM (comprising a guanidinium group) at physiological $\mathrm{pH}$, similarly to MGBG, this hypothesis should not be disregarded. Moreover, MGBG could also be transported in the unprotonated, uncharged form, since those transporters show an electroneutral behaviour (Grundemann et al. 2003).

Acknowledgments MPMM acknowledges financial support from the Portuguese Foundation for Science and Technology (FCT) Project PTDC/QUI/66701/2006 (co-financed by the European Community fund FEDER). Thanks are due to the Italian MIUR (Ministero dell'Istruzione, dell'Università e della Ricerca) and MIUR-PRINCofin (EA). Thanks are due to 'Fondazione Sovena' for the scholarship given to Nikenza Viceconte for supporting her Ph.D.

\section{References}

Agostinelli E, Arancia G, Dalla Vedova L, Belli F, Marra M, Salvi M, Toninello A (2004) The biological functions of polyamine oxidation products by amine oxidases: perspectives of clinical applications. Amino Acids 27:347-358

Agostinelli E, Belli F, Molinari A, Condello M, Palmigiani P, Dalla Vedova L, Marra M, Seiler N, Arancia G (2006) Toxicity of enzymatic oxidation products of spermine to human melanoma cells (M14): sensitisation by heat and MDL 72527. Biochim Biophys Acta 1763:1040-1050

Agostinelli E, Tempera G, Molinari A, Salvi M, Battaglia V, Toninello A, Arancia G (2007) The physiological role of biogenic amines redox reactions in mitochondria. New perspectives in cancer therapy. Amino Acids 33:175-187

Agostinelli E, Condello M, Molinari A, Tempera G, Viceconte N, Arancia G (2009) Cytotoxicity of spermine oxidation products to multidrug resistant melanoma cells (M14 ADR2): sensitisation by MDL 72527 , a lysosomotropic compound. Int $\mathrm{J}$ Oncol 35:485-498

Amado AM, Otero JC, Marques MPM, Batista de Carvalho LAE (2004) Spectroscopic and theoretical studies on solid 1, 2- ethylenediamine dihydrochloride salt. Chem Phys Chem 5:1837-1847

Amorim da Costa AM, Marques MPM, Batista de Carvalho LAE (2002) The carbon-hydrogen stretching region of the Raman Spectra of 1, 6-hexane-diamine: N-deuteration, ionization and temperature effects. J Vib Spec 29:61-67

Amorim da Costa AM, Marques MPM, Batista de Carvalho LAE (2003) Raman Spectra of putrescine, spermidine and spermine polyamines and their $\mathrm{N}$-deuterated and $\mathrm{N}$-ionised derivatives. J Raman Spec 34:357-366

Amorim da Costa AM, Batista de Carvalho LAE, Marques MPM (2004) Intra- vs. interchain interactions in $\alpha, \omega$-polyamines: a Raman Spectroscopy study. J Vib Spec 35:165-171

Averill-Bates DA, Cherif A, Agostinelli E, Tanel A, Fortier G (2005) Anti-tumoral effect of native and immobilized bovine serum amine oxidase in a mouse melanoma model. Biochem Pharmacol 69:1693-1704

Averill-Bates DA, Ke Q, Tanel A, Roy J, Fortier G, Agostinelli E (2008) Mechanism of cell death induced by spermine and amine oxidase in mouse melanoma cells. Int J Oncol 32:79-88

Babal P, Ruchko M, Campbell CC, Gilmour SP, Mitchell JL, Olson JW, Gillespie MN (2001) Regulation of ornithine decarboxylase activity and polyamine transport by agmatine in rat pulmonary artery endothelial cells. J Pharmacol Exp Ther 296:372-377

Bachrach U (2005) Naturally occurring polyamines: interaction with macromolecules. Curr Protein Pept Sci 6:559-566

Batista de Carvalho LAE, Lourenço LE, Marques MPM (1999) Conformational study of 1,2-diaminoethane by combined ab initio MO calculations and Raman spectroscopy. J Mol Struct 482 483: 639-646, and refs. therein

Battaglia V, Rossi CA, Colombatto S, Grillo MA, Toninello A (2007) Different behaviour of agmatine in liver mitochondria: inducer of oxidative stress or scavenger of reactive oxygen species? Biochim Biophys Acta 1768:1147-1153

Cabella C, Gardini G, Corpillo D, Testore G, Bedino S, Solinas SP, Cravanzola C, Vargiu C, Grillo MA, Colombatto S (2001) Transport and metabolism of agmatine in rat hepatocyte cultures. Eur J Biochem 268:940-947

Cardillo S, Iuliis AD, Battaglia V, Toninello A, Stevanato R, Vianello F (2009) Novel copper amine oxidase activity from rat liver mitochondria matrix. Arch Biochem Biophys 485:97-101

Carew JS, Nawrocki ST, Reddy VK, Bush D, Rehg JE, Goodwin A, Houghton JA, Casero RA, Marton LJ, Cleveland JL (2008) The novel polyamine analogue CGC-11093 enhances the antimyeloma activity of bortezomib. Cancer Res 68:4783-4790

Casero RA, Marton LJ (2007) Targeting polyamine metabolism and function in cancer and other hyperproliferative diseases. Nat Rev Drug Discov 6:373-390

Dalla Via L, Di Noto V, Siliprandi D, Toninello A (1996) Spermidine binding to liver mitochondria. Biochim Biophys Acta 1284:247252

Dudkowska M, Lai J, Gardini G, Stachurska A, GrzelakowskaSztabert B, Colombatto S, Manteuffel-Cymborowska M (2003) Agmatine modulates the in vivo biosynthesis and interconversion of polyamines and cell proliferation. Biochim Biophys Acta 1619:159-166

Fiuza SM, Amado AM, Oliveira PJ, Sardão VA, Batista de Carvalho LAE, Marques MPM (2006) Pt(II) vs Pd(II) Polyamine complexes as new anticancer drugs: a structure-activity study. Lett Drug Des Dev 3:149-151

Fogel-Petrovic M, Vujcic S, Miller J, Porter CW (1996) Differential post-transcriptional control of ornithine decarboxylase and spermidine-spermine N1-acetyltransferase by polyamines. FEBS Lett 391:89-94

Fogel-Petrovic M, Kramer DL, Vujcic S, Miller J, Mcmanis JS, Bergeron RJ, Porter CW (1997) Structural basis for differential 
induction of spermidine/spermine N1-acetyltransferase activity by novel spermine analogs. Mol Pharmacol 52:69-74

Gardini G, Cabella C, Cravanzola C, Vargiu C, Belliardo S, Testore G, Solinas SP, Toninello A, Grillo MA, Colombatto S (2001) Agmatine induces apoptosis in rat hepatocyte cultures. J Hepatol 35:482-489

Gerner EW, Meyskens FL Jr (2004) Polyamines and cancer: old molecules, new understanding. Nat Rev Cancer 4:781-792

Gerner EW, Meyskens FL Jr, Goldschmid S, Lance P, Pelot D (2007) Rationale for, and design of, a clinical trial targeting polyamine metabolism for colon cancer chemoprevention. Amino Acids 33:189-195

Goldenthal MJ, Marin-Garcia J (2004) Mitochondrial signaling pathways: a receiver/integrator organelle. Mol Cell Biochem 262:1-16

Grillo MA, Colombatto S (2004) Metabolism and function in animal tissues of agmatine, a biogenic amine formed from arginine. Amino Acids 26:3-8

Grillo MA, Battaglia V, Colombatto S, Rossi CA, Simonian AR, Salvi M, Khomutov AR, Toninello A (2007) Inhibition of agmatine transport in liver mitochondria by new charge-deficient agmatine analogues. Biochem Soc Trans 35:401-404

Grundemann D, Hahne C, Berkels R, Schomig E (2003) Agmatine is efficiently transported by non-neuronal monoamine transporters extraneuronal monoamine transporter (EMT) and organic cation transporter 2 (OCT2). J Pharmacol Exp Ther 304:810-817

Gupta R, Krause-Ihle T, Bergmann B, Muller IB, Khomutov AR, Muller S, Walter RD, Luersen K (2005) 3-Aminooxy-1aminopropane and derivatives have an antiproliferative effect on cultured Plasmodium falciparum by decreasing intracellular polyamine concentrations. Antimicrob Agents Chemother 49:2857-2864

Ha HC, Sirisoma NS, Kuppusamy P, Zweier JL, Woster PM, Casero RA (1998) The natural polyamine spermine functions directly as a free radical scavenger. Proc Natl Acad Sci USA 95:11140 11145

Harris A, Qu Y, Farrell N (2005) Unique cooperative binding interaction observed between a minor groove binding $\mathrm{Pt}$ antitumor agent and Hoechst dye 33258. Inorg Chem 44:11961198

Harris AL, Ryan JJ, Farrell N (2006) Biological consequences of trinuclear platinum complexes: comparison of [[trans$\mathrm{PtCl}(\mathrm{NH} 3) 2] 2 \mathrm{mu}-($ trans-Pt(NH3)2(H2N(CH2)6-NH2)2)]4+ (BBR 3464) with its non covalent congeners. Mol Pharmacol 69:666-672

Heby O, Persson L (1990) Molecular genetics of polyamine synthesis in eukaryotic cells. Trends Biochem Sci 15:153-158

Higashi K, Yoshida K, Nishimura K, Momiyama E, Kashiwagi K, Matsufuji S, Shirahata A, Igarashi K (2004) Structural and functional relationship among diamines in terms of inhibition of cell growth. J Biochem 136:533-539

Huang Y, Keen JC, Pledgie A, Marton LJ, Zhu T, Sukumar A, Park BH, Blair B, Brenner K, Casero RA, Davidson NE (2006) Polyamine analogues down-regulate estrogen receptor alpha expression in human breast cancer cells. J Biol Chem 281:19055-19063

Ichimura S, Hamana K, Nenoi M (1998) Significant increases in the steady states of putrescine and spermidine/spermine N1-acetyltransferase mRNA in HeLa cells accompanied by growth arrest. Biochem Biophys Res Commun 243:518-521

Jänne J, Alhonen L, Leinonen P (1991) Polyamines: from molecular biology to clinical applications. Ann Med 23:241-259

Komeda S, Moulaei T, Woods KK, Chikuma M, Farrell NP, Williams LD (2006) A third mode of DNA binding: phosphate clamps by a polynuclear platinum complex. J Am Chem Soc 128:1609216103
Li G, Regunathan S, Barrow CJ, Eshraghi J, Cooper R, Reis DJ (1994) Agmatine: an endogenous clonidine-displacing substance in the brain. Science 263:966-969

Liu Q, Qu Y, Van Antwerpen R, Farrell N (2006) Mechanism of the membrane interaction of polynuclear platinum anticancer agents. Implications for cellular uptake. Biochemistry 45:4248-4256

Lortie MJ, Novotny WF, Pederson OW, Vallon V, Malvey K, Mendonca M, Satriano J, Insel P, Thomson SC, Blantz RC (1996) Agmatine, a bioactive metabolite of arginine. Production, degradation, and functional effects in the kidney of the rat. J Clin Invest 97:413-420

Manni A, Washington S, Hu X, Griffith JW, Bruggeman R, Demers LM, Mauger D, Verderame MF (2006) Effects of polyamine synthesis inhibitors on primary tumor features and metastatic capacity of human breast cancer cells. Clin Exp Metastasis 22:255-263

Marques MPM, Batista de Carvalho LAE (2000) COST 917: Biogenically active amines in food. In: Morgan DML, White A, Sánchez-Jiménez F, Bardocz S (eds) Theoretical approach to the conformational preferences of putrescine. European commission, Luxembourg, p 122

Marques MPM, Batista de Carvalho LAE (2007) Vibrational spectroscopy studies on linear polyamines. Biochem Soc Trans 35:374-380

Marques MPM, Batista de Carvalho LAE, Tomkinson J (2002a) Study of biogenic and $\alpha, \omega$-polyamines by combined inelastic neutron scattering and Raman spectroscopies, and ab initio MO calculations. J Phys Chem A 106:2473-2482

Marques MPM, Girão T, Pedroso de Lima MC, Gameiro A, Pereira E, Garcia P (2002b) Cytotoxic effects of metal complexes of biogenic polyamines. I-Platinum(II) spermidine compounds: Prediction of their antitumour activity. Biochim Biophys Acta 1589:63-70

Marques MPM, Batista de Carvalho LAE, Tomkinson J (2006) Transverse acoustic modes of biogenic and alpha, omegapolyamines: a study by inelastic neutron scattering and Raman spectroscopies coupled to DFT calculations. J Phys Chem 110:12947-12954

Marques MPM, Borges F, Amorim da Costa AM, Batista de Carvalho LAE (2007) Vibrational spectroscopy studies on biologically relevant molecules. From anticancer agents to drugs of abuse. In: Kneip K, Aroca R, Kneipp H, Wentrup-Byrne E (eds) ACS symposium \$eries no. 963-new approaches in biomedical spectroscopy. American Chemical Society, Washington, DC, pp 338-363

Marques MPM, Gil FPSC, Calheiros R, Battaglia V, Brunati AM, Toninello A (2008) Biological activity of antitumoural MGBGthe structural variable. Amino Acids 34:555-564

Marton LJ, Pegg AE (1995) Polyamines as targets for therapeutic intervention. Annu Rev Pharmacol Toxicol 35:55-91

N'soukpo-Kossi CN, Ouameur AA, Thomas T, Shirahata A, Thomas TJ, Tajmir-Riahi HA (2008) DNA interaction with antitumor polyamine analogues: a comparison with biogenic polyamines. Biomacromolecules 9:2712-2718

Nakaike S, Kashiwagi K, Terao K, Iio K, Igarashi K (1988) Combined use of alpha-difluoromethylornithine and an inhibitor of S-adenosylmethionine decarboxylase in mice bearing P388 leukaemia or Lewis lung carcinoma. Jpn J Cancer Res 79:501508

Nasizadeh S, Myhre L, Thiman L, Alm K, Oredsson S, Persson L (2005) Importance of polyamines in cell cycle kinetics as studied in a transgenic system. Exp Cell Res 308:254-264

Ouameur AA, Tajmir-Riahi HA (2004) Structural analysis of DNA interactions with biogenic polyamines and cobalt(III)hexamine studied by Fourier transform infrared and capillary electrophoresis. J Biol Chem 279:42041-42054 
Patel MM, Anchoroquy TJ (2006) Ability of spermine to differentiate between DNA sequences-preferential stabilization of A-tracts. Biophys Chem 122:5-15

Pegg AE (2008) Spermidine/spermine-N(1)-acetyltransferase: a key metabolic regulator. Am J Physiol Endocrinol Metab 294:E995E1010

Pegg AE, Feith DJ (2007) Polyamines and neoplastic growth. Biochem Soc Trans 35:295-299

Pezzato E, Battaglia V, Brunati AM, Agostinelli E, Toninello A (2009) $\mathrm{Ca}^{2+}$-independent effects of spermine on pyruvate dehydrogenase complex activity in energized rat liver mitochondria incubated in the absence of exogenous $\mathrm{Ca}^{2+}$ and $\mathrm{Mg}^{2+}$. Amino Acids 36:449-456

Pleshkewych A, Kramer DL, Kelly E, Porter CW (1980) Independence of drug action on mitochondria and polyamines in L1210 leukemia cells treated with methylglyoxal-bis(guanylhydrazone). Cancer Res 40:4533-4540

Pratesi G, Perego P, Polizzi D, Righetti SC, Supino R, Caserini C, Manzotti C, Giuliani FC, Pezzoni G, Tognella S, Spinelli S, Farrell N, Zunino F (1999) A novel charged trinuclear platinum complex effective against cisplatin-resistant tumours: hypersensitivity of p53-mutant human tumour xenografts. Br J Cancer 80:1912-1919

Qu Y, Rauter H, Soares-Fontes AP, Bandarage R, Kelland LR, Farrell N (2000) Synthesis, characterization and cytotoxicity of trifunctional dinuclear platinum complexes: comparison of effects of geometry and polyfunctionality on biological activity. J Med Chem 43:89-92

Raasch W, Schafer U, Chun J, Dominiak P (2001) Biological significance of agmatine, an endogenous ligand at imidazoline binding sites. Br J Pharmacol 133:755-780

Regenass U, Caravatti G, Mett H, Stanek J, Schneider P, Muller M (1992) New S-adenosylmethionine decarboxylase inhibitors with potent antitumor activity. Cancer Res 52:4712-4718

Ruiz-Chica J, Medina MA, Sanchez-Jimenez F, Ramirez FJ (2003) Raman Spectroscopy study of the interaction between biogenic polyamines and an alternating AT oligodeoxyribonucleotide. Biochim Biophys Acta 1628:11-21

Salvi M, Battaglia V, Mancon M, Colombatto S, Cravanzola C, Calheiros R, Marques MP, Grillo MA, Toninello A (2006) Agmatine is transported into liver mitochondria by a specific electrophoretic mechanism. Biochem J 396:337-345

Satriano J, Matsufuji S, Murakami Y, Lortie MJ, Schwartz D, Kelly CJ, Hayashi S, Blantz RC (1998) Agmatine suppresses proliferation by frameshift induction of antizyme and attenuation of cellular polyamine levels. J Biol Chem 273:15313-15316

Satriano J, Kelly CJ, Blantz RC (1999) An emerging role for agmatine. Kidney Int 56:1252-1253
Sava IG, Battaglia V, Rossi CA, Salvi M, Toninello A (2006) Free radical scavenging action of the natural polyamine spermine in rat liver mitochondria. Free Radic Biol Med 41:1272-1281

Scalabrino G, Ferioli ME (1981) Polyamines in mammalian tumors. Part I. Adv Cancer Res 35:151-268

Scalabrino G, Ferioli ME (1982) Polyamines in mammalian tumors. Part II. Adv Cancer Res 36:1-102

Schipper RG, Penning LC, Verhofstad AA (2000) Involvement of polyamines in apoptosis. Facts and controversies: effectors or protectors? Semin Cancer Biol 10:55-68

Seiler N, Raul F (2005) Polyamines and apoptosis. J Cell Mol Med 9:623-642

Simonian AR, Grigorenko NA, Vepsalainen J, Khomutov AR (2005) New charge-deficient agmatine analogs. Bioorg Khim 31:583587

Teixeira LJ, Seabra M, Reis E, Girão da Cruz MT, Pedroso de Lima MC, Pereira E, Miranda MA, Marques MPM (2004) Cytotoxic activity of metal complexes of biogenic polyamines: polynuclear platinum(II). J Med Chem 47:2917-2925

Todd BA, Parsegian VA, Shirahata A, Thomas TJ, Rau DC (2008) Attractive forces between cation condensed DNA double helices. Biophys J 94:4775-4782

Toninello A, Salvi M, Mondovì B (2004) Interaction of biologically active amines with mitochondria and their role in the mitochondrial-mediated pathway of apoptosis. Curr Med Chem 11:23492374

Toninello A, Battaglia V, Salvi M, Calheiros R, Marques MPM (2006) Structural characterisation of agmatine at physiological conditions. Struct Chem 17:163-175

Vargiu C, Cabella C, Belliardo S, Cravanzola C, Grillo MA, Colombatto S (1999) Agmatine modulates polyamine content in hepatocytes by inducing spermidine/spermine acetyltransferase. Eur J Biochem 259:933-938

Von Hoff DD (1994) MGBG: teaching an old drug new tricks. Ann Oncol 5:487-493

Wallace HM (2003) Polyamines and their role in human disease-an introduction. Biochem Soc Trans 31:354-355

Wallace HM (2007) Targeting polyamine metabolism: a viable therapeutic/preventative solution for cancer? Expert Opin Pharmacother 8:2109-2116

Wallace HM, Fraser AV, Hughes A (2003) A perspective of polyamine metabolism. Biochem J 376:1-14

Williams-Ashman HG, Schenone A (1972) Methyl glyoxal bis(guanylhydrazone) as a potent inhibitor of mammalian and yeast $\mathrm{S}$ adenosylmethionine decarboxylases. Biochem Biophys Res Commun 46:288-295 